\title{
Application of Phase Doppler Interferometry and Ultra Small Angle $X$-ray Scattering to the Near Field Spray from a Reference Simplex Atomizer
}

\author{
Scott Leask ${ }^{1}$, Vincent McDonell ${ }^{1, *}$ \\ ${ }^{1}$ UCI Combustion Laboratory, University of California, Irvine CA, USA \\ Brandon A. Sforzo ${ }^{2}$, Alan L. Kastengren ${ }^{2}$, Jan llavsky ${ }^{2}$, Christopher F. Powell ${ }^{2}$ \\ ${ }^{2}$ Argonne National Laboratory, 9700 S. Cass Ave, Lemont, IL 60439, USA \\ ${ }^{*}$ Corresponding author: mcdonell@ucicl.uci.edu
}

\begin{abstract}
This effort continues the utilization of a reference simplex atomizer spray as a means to facilitate comparison between diagnostics and laboratories. In this work, focus is on comparison of two methods that can provide information associated with droplet sizes. The near field (e.g., within $10 \mathrm{~mm}$ of the injector) is interrogated. To provide boundary conditions for Lagrangian type CFD simulations, phase Doppler interferometry is commonly applied. In this case, measurements are sought "as close as possible" to the injector. This creates a measurement dilemma in that the presence of ligaments or non-spherical droplets can result in much of the mass from being measured. As a result, some bias may exist towards smaller droplets which will tend to become spherical (and thus validated by the signal processing logic) quicker than larger droplets. As a potential alternate or compliment to the traditional PDI measurements of the droplet sizes and velocities, Ultra-Small Angle X-Ray Scattering (USAXS) can also be used to quantify details regarding the degree of atomization. Direct measurements of interfacial surface area of the spray is combined with radiography of the liquid volume to determine droplet size information. This technique is well suited to probe optically thick regions of the spray where PDI may have challenges with multiple particles in the measurement volume. Additionally, USAXS has ability to measure relatively fine particles, even those below 1 micron in diameter, complementing PDI suitability for measurement of droplet sizes larger than 1 micron. In this paper, a reference spray generated by two builds of a standard pressure swirl atomizer is used to create sprays at different injection pressures. It is noted that one of the builds generates a spray "defect" at the lower pressure studied $(345 \mathrm{kPa})$. In general, the results illustrate how the methods can complement each other and provide even more information about the spray in the near field. For comparison, the $10 \mathrm{~mm}$ axial location is used. As the PDI and USAXS measurements rely on completely different measurement principles, considerable manipulation of the measurements are required to provide a direct comparison. For the current study, emphasis was placed on manipulating the PDI point measurements into corresponding projected line averages that are inherent to the USAXS and radiography results. General agreement in trends and magnitude is observed for the projected mass measured by both methods. Furthermore, excellent agreement is obtained for the droplet SMD values except for the spray with the defect at $345 \mathrm{kPa}$.
\end{abstract}

\section{Keywords}

PDI, X-ray, Diagnostics, USAXS, Simplex, Pressure-swirl

\section{Introduction}

Identifying droplet characteristics and measuring or predicting the droplet size of fuel sprays is fundamental to the advancement of numerous applications in combustion systems, process industries, agriculture, medicine, and meteorology [1]. To date, droplet sizing techniques, such as phase Doppler interferometry (PDI) and laser diffraction, provide information that is vital to such applications. Yet data in the immediate near field of atomizers are challenging to obtain due to non-spherical droplets and potentially high optical thickness. Another promising method for obtaining drop size is Ultra-small-angle x-ray scattering (USAXS) [2, 3]. This method determines drop size in a much different manner than does PDI. Comparison and correlation of data obtained from differing diagnostic methods and simulations across and within institutions is desired to help provide confidence in each method as well as to provide comprehensive insight necessary to further enhance fuel spray technology through simulations and innovation. In the case that data are collected from non-identical diagnostics, misinterpretation of data may arise due to differences in, for example, instrumentation design [4] and user protocol [5]. Therefore, a need for a "standard spray" [6] for comparison of diagnostics and modeling and thus nonbiased data interpretation is evident. The effectiveness of this approach has been illustrated via the "standard sprays" established to represent diesel injection by the Engine Combustion Network (ECN) [7]. The atomizer producing the standard spray must demonstrate high reproducibility and minor variation in the spray produced in order to be used with various researchers and diagnostics. Although previous efforts [6] had modest success in establishing a standard simplex spray, recently a new version of this device has been introduced [8]. This new version is fully supported and maintained by Advanced Atomization Technologies (AAT) which is a joint venture between GE and Parker Aerospace. Figure 1 illustrates a cross section of this atomizer. To date 10 builds of this particular reference spray are available for users. This particular atomizer represents one class of injector found in wide ranging applications including combustion systems such as aeroengines and liquid fueled heaters. 


\section{Objective}

The objective of the present work is to directly compare, for the first time, droplet size and concentration measurements obtained by two diagnostic methods, PDI and USAXS. Results were obtained at two independent laboratory facilities, UC Irvine (PDI) and Argonne National Laboratory (USAXS). The utility of the standard spray in this case is evident as it was far more convenient to exchange atomizers than to relocate the measurement equipment. Neither facility had access to both diagnostics at their respective sites. To better assess the comparison, two builds were studied, SN005 and SN010. In addition to comparing the diagnostics, the data obtained represent additional information about the behavior of the spray produced by these atomizers that can serve as a basic for insight and validation data for simulations.

\section{Methodology}

Two Research Simplex Atomizer injectors and they spray they produced were studied. These injectors, produced for spray and combustion studies with practical industry methods and materials, named "SN005" and "SN010" have been compared and analyzed previously $[8,9,10]$. As in these previous studies, the injectors were supplied with water via a dip-tube tank, pressurized using gaseous nitrogen. The atomizers were also mounted such that the spray injected downward to replicate the conditions and methods carried out in the prior studies. Here, the supplied fluid pressure was set to either $345 \mathrm{kPa}(50 \mathrm{psi})$ or $690 \mathrm{kPa}(100 \mathrm{psi})$ as measured immediately upstream of the injector housing. The injector design, as tested here, is presented in Figure 1, to illustrate the coordinate convention.

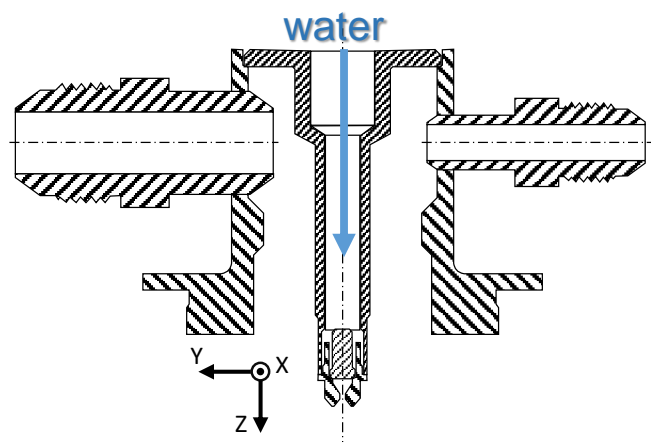

Figure 1. Coordinate system of simplex atomizers.

\section{Phase Doppler Interferometry}

For PDI, data were obtained at UC Irvine (UCI) using a two-component TSI Phase Doppler Particle Analyzer (PDPA) with a FSA 4000 processor to process signals associated with point measurements of droplet velocities and sizes. A Fiberlight multicolor beam separator was used to output both unshifted and shifted beams of $514 \mu \mathrm{m}$ and $488 \mu \mathrm{m}$ wavelengths into a fiber optic coupled two component transmitter. The PDPA receiver was positioned to collect scattered light 30 degrees off forward from the two component transmitter line of sight. The rough arrangement of equipment is depicted in Figure 2 The details of the optical settings and corresponding droplet size and velocity ranges used are summarized in Leask et al. [8]

\section{Radiography}

The diagnostic layout for X-ray radiography measurements collected at the 7-BM beamline of the Advanced Photon Source (APS) at Argonne National Laboratory are thoroughly described in previous works [11, 12]. Specifically here, the synchrotron-produced $\mathrm{X}$-ray light was conditioned to $8.0 \pm 0.01 \mathrm{keV}$ mean photon energy $(4 \% \Delta \mathrm{E} / \mathrm{E})$ and focused to a waist of $4 \times 6 \mu \mathrm{m}(\mathrm{V} \times \mathrm{H})$ located at the tip of the injector as the experimental origin. After transmission through the spray region of interest, the beam intensity, $I$, was measured with a PIN diode and sampled to $3.68 \mu$ mtime resolution. At each spatial location, the time history was recorded to allow for spectral analysis. Following the Beer-Lambert law, the projected mass of the spray $(M)$ within the X-ray path was computed from the beam intensity measurements as described by:

$$
M(x, y, \theta, t)=-\frac{1}{\mu} \log \left[\frac{I(x, y, \theta, t)}{I_{0}(t)}\right]
$$

where $\mu$ is the mass attenuation coefficient, collected empirically and compared to well-established quantities for water. The parameters $I_{0}(t)$ and $I(x, y, \theta, t)$ are the measured beam intensities of the incident beam and the transmitted beam, respectively.

\section{Ultra-small-angle $X$-ray Scattering}

USAXS measurements were carried out at the 9-ID beamline [13] of the APS, using a Bonse-Hart instrument as schematized in Figure 3. In this configuration, the incoming X-ray light has been conditioned to a photon energy 


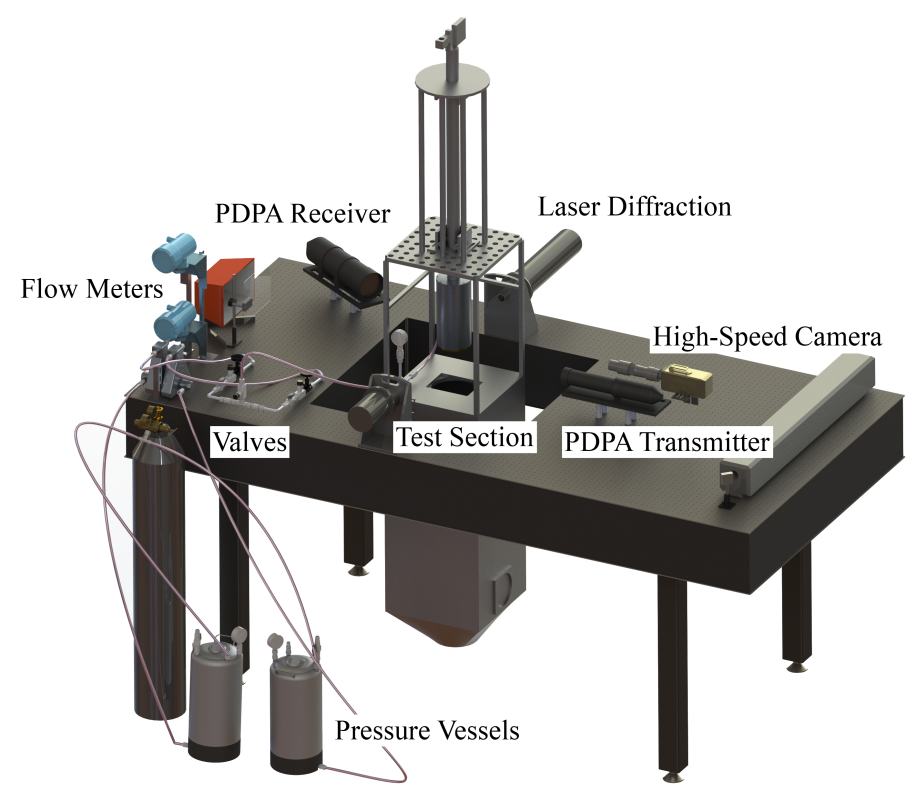

Figure 2. Experimental setup of the spray and PDI system at UCI.

of $21 \mathrm{keV}(\lambda=0.59 \AA)$, and profiled to $200 \times 200 \mu \mathrm{m}(\mathrm{V} \times \mathrm{H})$ with beam-defining slits. The X-rays are precisely collimated with pair of $\mathrm{Si}(220)$ crystals and the beam intensity is measured with an ion chamber. The beam then passes into the spray, scattering the photons at small angles.

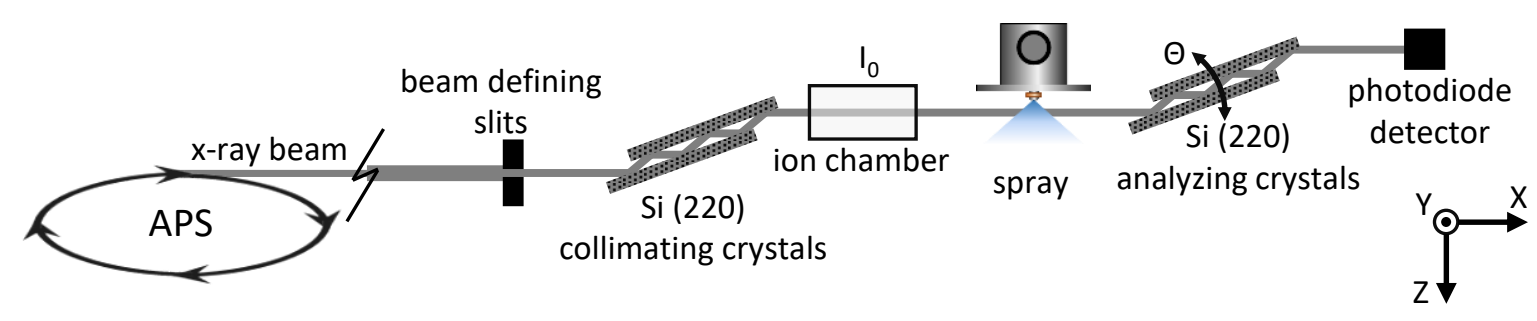

Figure 3. Layout of the USAXS experiment.

The scattered x-ray intensity as a function of angle is converted to differential cross section $(d \Sigma / d \Omega)$ as a function of scattering vector $(q)$. Due to the fact that the droplet sizes are much larger than the smallest measurable scattering vector with the USAXS instrument, a scattering curve can be constructed and Porod's law [14] exploited to relate to the path-specific surface area of the probed spray. This relationship assumes that the particles are homogeneous, relatively dilute, and locally non-interacting. Furthermore, it is assumed that the particles are randomly oriented which is expected to hold for this water spray after the sheet breakup region where comparisons are made to the PDI measurements.

Radiography derived projected mass measurements, in combination with the water density, result in liquid volume. Assuming that the spray consists of spherical droplets, this liquid volume is combined with the USAXS $S_{p}$ values from common measurement locations to yield the Sauter mean diameter (SMD, $d_{32}$ ), via the established formulation $[2,15]$ of Eq. 2.

$$
D_{32}=6 \frac{V}{S_{p}},
$$

\section{Results and discussion}

Before getting into the comparison of the diagnostic measurements, it is important to note that these methods are vastly different relative to their measurement principles. PDI uses an interferometric sample volume and fundamentally measures the size and velocity of spherical particles using refracted light at discrete points. USAXS is a line of sight method using scattering, and for our case, fundamentally measures the interfacial surface area due to sharp density gradients in the probe beam. As a result, considerable manipulation of the fundamental results obtained is needed to compare the measurements. In the present case, the PDI data have been converted into equivalent line averaged data. This involves combining results from individual points along a line. Because PDI does not necessarily measure for the same time at each point, that must be considered. PDI samples all droplets passing through 
the interferometric sample volume. USAXS gathers scattered photons from particles within the beam path. As a result, slower moving particles will inherently scatter more photons. To account for this, the PDI size data can also be weighted by the particle velocities. Is the subsequent sections, the steps involved in the various manipulations are noted.

\section{Mass Distribution}

For comparison to X-ray spray tomography results, the spray density $\left(\rho_{\mathrm{s}}\right)$ were computed from the PDI droplet statistic summary values via the following relation:

$$
\rho_{\mathrm{s}}=n \frac{\pi D_{30}^{3}}{6},
$$

where, $n$ is the measurement particle concentration in counts per volume and $D_{30}$ is the volume mean diameter of measured size distribution. This calculation is performed for each of the 100+ discrete PDI measurement locations at the $10 \mathrm{~mm}$ axial location and the mapping of $\rho_{\mathrm{s}}$ for each injector and condition is presented in Figure 4. These illustrate both that the time-averaged spray extent and asymmetry are higher for the $345 \mathrm{kPa}$ cases. This is consistent with spray density mappings from $\mathrm{x}$-ray spray tomography presented previously [9]. Furthermore, the peak spray densities in those profiles were on the order of $400 \mu \mathrm{g} / \mathrm{mm}^{3}$ at an axial distance of $0.2 \mathrm{~mm}$ from the nozzle exit. These peak values are expected to decrease precipitously as the spray broadens and spreads, which is illustrated in this presentation of the PDI data.
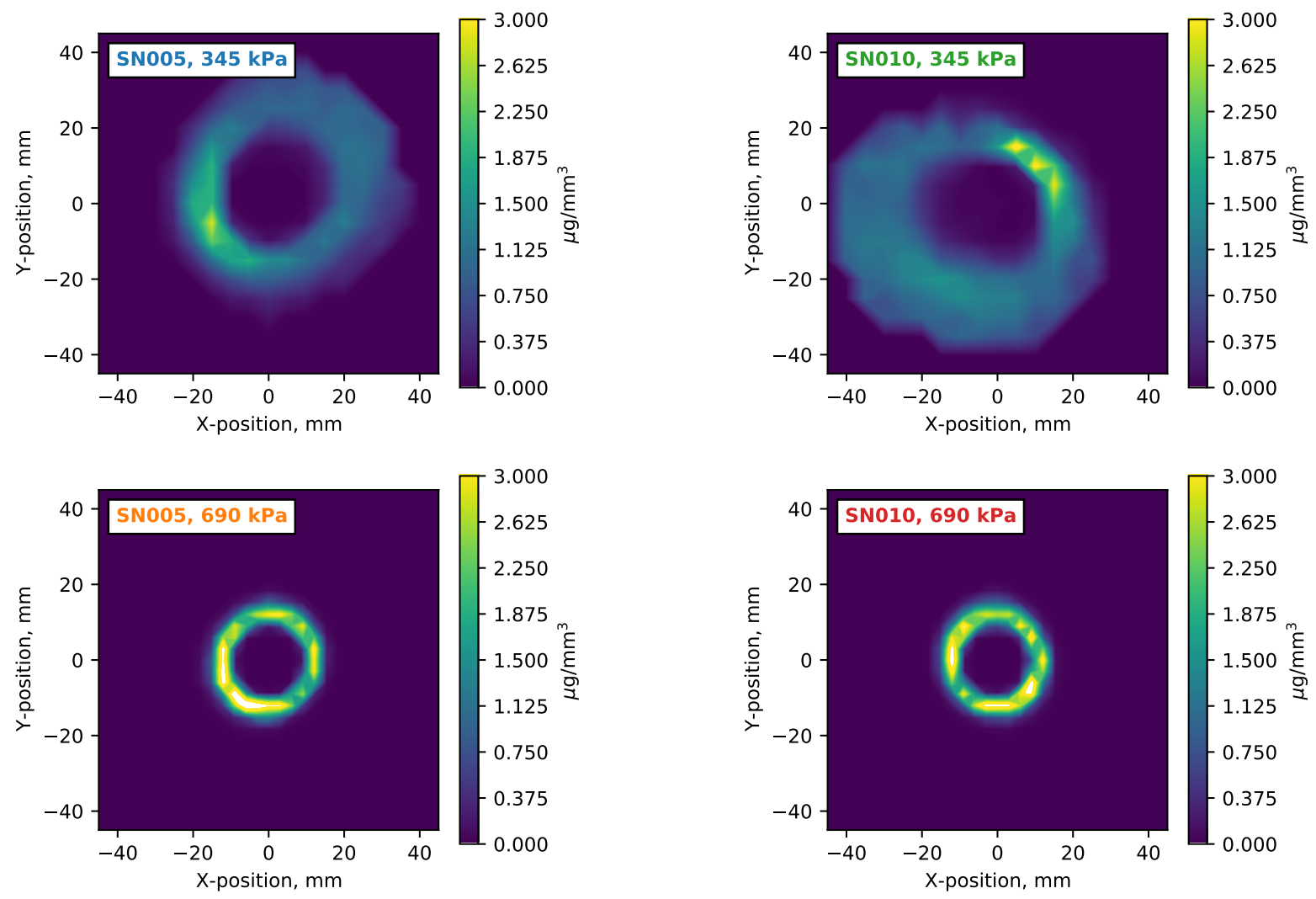

Figure 4. Planar maps of the liquid mass per volume measurements calculated using "Method 2" from PDI measurements.

For further comparison to x-ray radiography results at common spatial locations the PDI spray density mappings were integrated along the line-of-sight dimension to produce projected mass $(M)$ profiles. These profiles for each condition are plotted in Figure 5.

The results show the expected general trend with paths through the region of the spray with the highest mass (recall Figure 4) having higher peak values. The path through $Y=0$ actually traverses thinner regions of peak mass and thus show a dip in the profile. These trends are similar for both nozzles and both conditions. The narrower radial extent at the higher pressure case is expected as well as the atomizers attain full atomization at $690 \mathrm{kPa}$. At $345 \mathrm{kPa}$, the sprays are less symmetric. At the $Y=0$ position, the corresponding radiography based projected mass is shown in the solid symbols. While the values are systematically lower than the PDI, they are of same order of magnitude. Again, the PDI results have been manipulated to integrate them along the path and to weight them by data rate and combine the measurement of concentration with the size. The measurement of concentration involves 


\begin{tabular}{llll}
\hline Injector & injection pressure, $\mathrm{kPa}$ & $\mathrm{TIM}_{\mathrm{x}-\text { ray }}, \mu \mathrm{g} / \mathrm{mm}$ & PIM $_{\text {PDI }}, \mu \mathrm{g} / \mathrm{mm}$ \\
\hline \hline SN005 & 345 & 533.3 & 2223.2 \\
SN005 & 690 & 320.2 & 1005.1 \\
SN010 & 345 & 630.7 & 2999.2 \\
SN010 & 690 & 315.6 & 988.6 \\
\hline
\end{tabular}

Table 1. Integrated mass values for each condition as calculated from the $x$-ray radiography scan $\left(\mathrm{TIM}_{\mathrm{x}-\mathrm{ray}}\right)$ at $Z=0.2 \mathrm{~mm}$, and the PDI planar measurements (PIMPDI) at $Z=10.0 \mathrm{~mm}$.

accurate counting of droplets and knowledge of the sample volume dimensions. In essence, a number of different direct and indirect measurements are combined. Given this, that the two methods give results within the same of order of magnitude variation is considered reasonable.
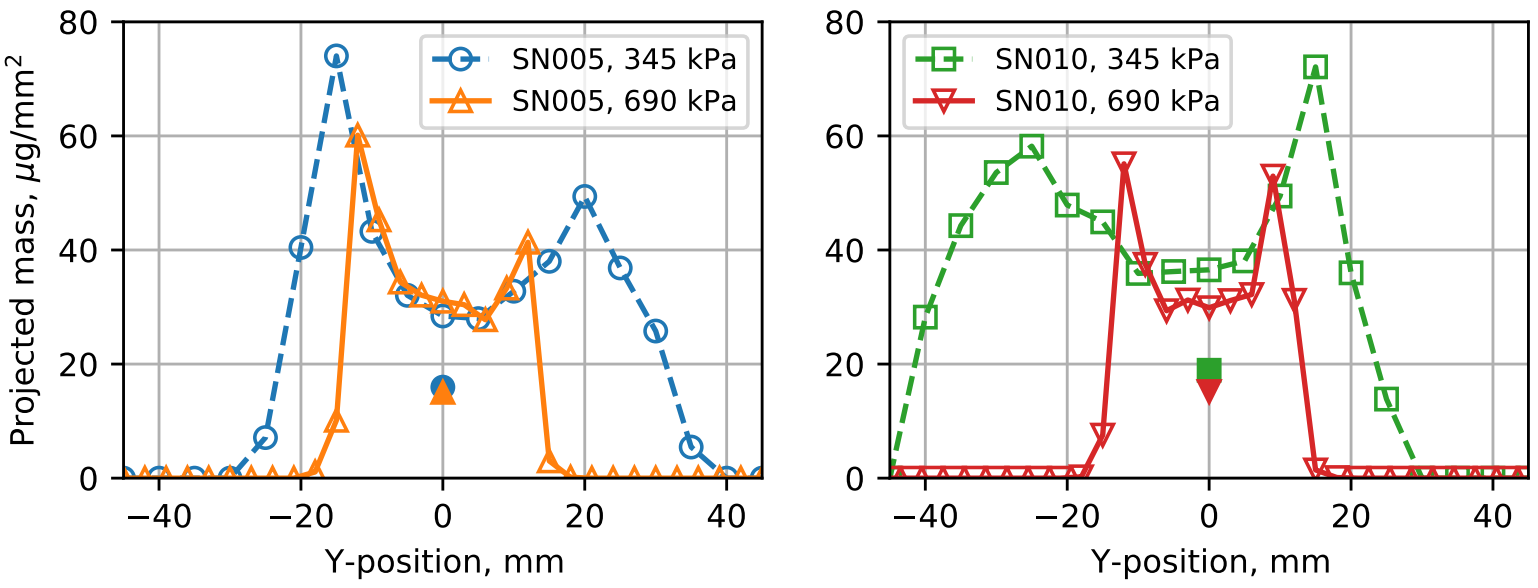

Figure 5. Transverse scans of projected mass calculated from PDI measurements for SN005 (left) and SN010 (right). Values depicted by solid symbols are corresponding projected mass from $\mathrm{x}$-ray radiography

Note that, while the PDI measurements can directly measure the velocities of droplets through the probe volume, the $\mathrm{x}$-ray projected mass measurements provide the only way to quantitatively assess the flow of mass through the region of interest with $\mathrm{x}$-rays. The integration of this projected mass can be compared at the various downstream distances for each of the conditions that radiography was collected. This transverse integrated mass (TIM) was computed using Eq. 4 for each axial distance (Z).

$$
\operatorname{TIM}(z)=\int M(y, z) d y
$$

In the computation for TIM, the beam projected probe volume provides the integration along the line of sight. Analogous to this process is spatially integrating the spray density derived from the PDI measurements, according to Eq. 5 in a planar integrated mass (PIM).

$$
\operatorname{PIM}(z)=\iint \rho_{\mathrm{s}}(x, y, z) d y d x
$$

Though one spatial integration is performed physically, the values for TIM and PIM should be comparable for the sake of tracking the mass in the region of interest. These values for each diagnostic are presented in Table 1.

By conservation of mass, each transverse or planar slice should provide comparable values but for the duration that the liquid mass remains within the measurement probe. Therefore, the evolution of TIM in the direction of the spray, or from condition to condition, relates to the velocity of the liquid via Eq. 6 . This indicates, through Table 1, that for increasing injection pressure, the nominal TIM value decreases, indicating the higher relative mean velocity of the fluid. This also indicates, that for the measurements at $345 \mathrm{kPa}$, the mean velocity of the fluid was higher for SN005 than SN010. When compared to PIM values from PDI downstream, the absolute values for both $690 \mathrm{kPa}$ cases are very similar, indicating a consistent flow through both injectors at this condition. However, the evolution from TIM to PIM suggests there is a significant deceleration of the liquid droplets. In each of the $345 \mathrm{kPa}$ cases, there is a $77 \%$ decreases, while in each of the $690 \mathrm{kPa}$ cases, there is only a $68 \%$ decrease. Based on the initial TIM values, the 
higher velocities (and lower dispersion) in the $690 \mathrm{kPa}$ case likely contribute to the ability for droplets to sustain their momentum.

$$
\frac{v_{m a}}{v_{r e f}}=\frac{\mathrm{TIM}_{r e f}}{\mathrm{TIM}}
$$

\section{Atomization Performance}

Figure 6 presents the comparison of the SMD from the two instruments. The results are arranged for each atomizer (SN005 and SN010). The open symbols represent the path averaged PDI results. The majority of the results are for the data rate weighted PDI measured SMD. In addition, at $Y=0$, the star symbols shows the results for velocity weighted SMD. Recall that droplets spending more time in the sample volume of the USAXS beam line will scatter proportionally more energy than those spending less time. Hence these star symbols capture that effect. Finally, the solid symbols represent the line integrated results obtained from USAXS. With the exception of the $345 \mathrm{kPa}$ spray for the SN005 case, the SMD values exhibit good agreement with values in the 100-125 $\mu$ mrange. These results are arguably within the experimental uncertainty of the instruments. For the SN005 atomizer at $345 \mathrm{kPa}$, it is believed that the dynamic behavior of the spray at this condition creates challenges for baselining the USAXS measurements and contributes to variability overall and thus should not be considered to be representative.

$$
D_{32}(y)=\frac{\sum_{x} D_{32}(x, y) f_{\text {data }}(x, y) / f_{\text {data }, \max }(y)}{\sum_{x} f_{\text {data }}(x, y) / f_{\text {data } \max }(y)},
$$

where, $f_{\text {data }}$ is the PDI acquisition data rate, and $f_{\text {data,max }}$ is the maximum value along the line of sight being summed.
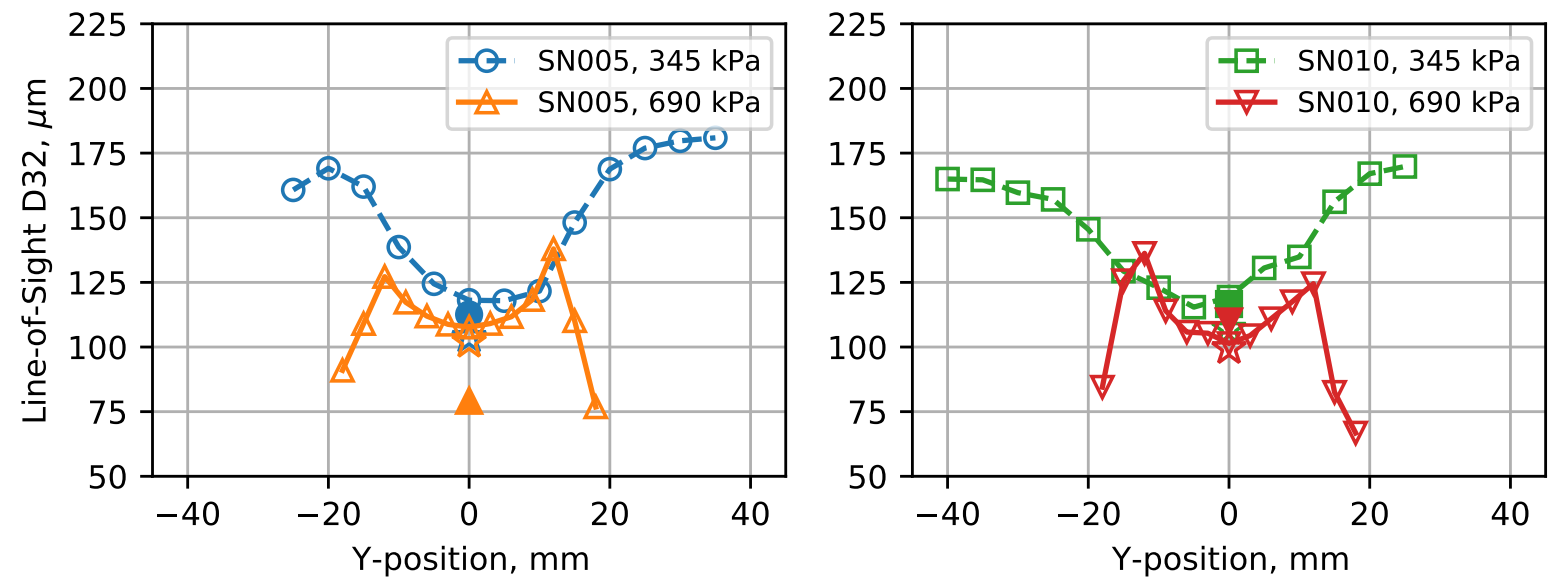

Figure 6. Line-of-sight probed Sauter Mean Diameter for SN005 (left) and SN010 (right). Open star symbols represent velocity corrected PDI measurements of SMD. Values depicted by solid symbols are corresponding SMD from combined X-ray radiography and USAXS measurements.

The current comparison of SMD between diagnostics suffers from limited radiography data at the $Z=10 \mathrm{~mm}$ plane to compute $D_{32}$ via Eq. 2 . Though radiography collection focused closer to the nozzle, a more comprehensive mapping of the spray was gathered with USAXS, including full transverse scans at $Z=10 \mathrm{~mm}$. These USAXS scans directly provide quantification for the path-specific surface area of the droplets in the spray, and are presented as the solid markers in Figure 7. The erratic behavior of injector SN005, particularly at the $345 \mathrm{kPa}$ condition is reflected in the very asymmetric profile of the scattering.

Two methods were used to compute comparable $S_{p}$ values using PDI data. Firstly, a reverse calculation of liquid volume through Eq. 2 using the profiles of data-rate weighted SMD and projected mass was calculated. These values are labeled as "PDI:M/D32" in Figure 7, represented by open markers, and generally over-predict the $S_{p}$ when compared to the USAXS values.

Next, an analog to the mass concentration computation in Eq. 3, which used the volume mean diameter $\left(D_{30}\right)$, was produced for computing the surface area from the surface mean diameter $\left(D_{20}\right)$, and is presented in Eq. 8.

$$
S_{p}(y)=\sum_{x} n \pi D_{20}^{2}
$$


These projected values of $S_{p}$ are represented as the half filled symbols in each of the plots in Figure 7, are labeled "PDI:D20," and generally fall below the USAXS produced $S_{p}$ values. Considering the methods that were used to compute the path-specific surface area from the PDI data, production of values that are along the same order of magnitude as the direct measurement from USAXS is positive. The PDI-produced profiles generally bound the USAXS profiles, and also reflect similar asymmetries, specifically in the SN005, $690 \mathrm{kPa}$ and the SN010, $345 \mathrm{kPa}$ case. Lastly, progressing downstream, the evolution of the USAXS profiles has shown order of magnitude change in $S_{p}$, particularly along the edges (line-of-sight tangent), and asymptotic nature beyond the region of sheet disintegration. The scattering from the centerline of the spray is more monotonic and therefore provides a better point of comparison, which relates more closely to the $S_{p}$ values computed via Eq. 8.
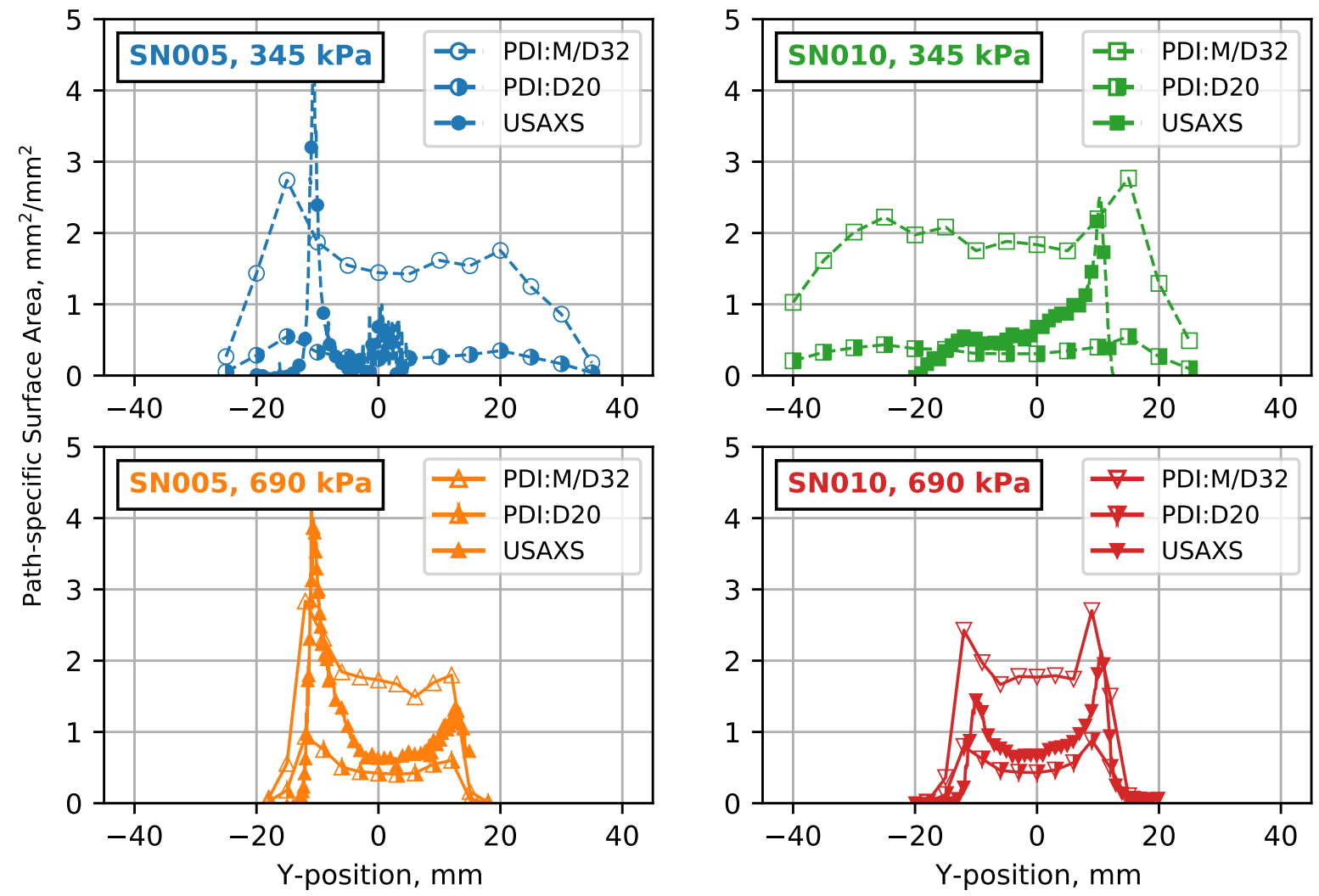

Figure 7. Path-specific Surface Area $\left(S_{p}\right)$ for SN005 (left) and SN010 (right). Open symbols represent $S_{p}$ determined from PDI SMD and projected mass. Half-filled symbols represent $S_{p}$ determined from concentration weighted $D_{20}$ integration. Values depicted by solid symbols are corresponding $S_{p}$ collected from USAXS measurements.

\section{Summary and Conclusions}

In this paper, various comparisons between PDI and USAXS have been carried out for two builds of a standard research simplex atomizer. The results provide numerous insights into the overall spray structure and comparisons between the measurement methods.

A critical observation is that SN005 exhibits some spray defects that are manifested at the $345 \mathrm{kPa}$ condition. This is much less noticeable at $690 \mathrm{kPa}$. However, the defects at $345 \mathrm{kPa}$ result in dynamic spray spatially which leads to measurement challenges for both methods. Measurements by both methods demonstrate more asymmetry for the SN005 than for SN010 at the lower pressure condition. Yet despite these fundamental differences, the current work indicates a high degree of correspondence between the measured results. For example:

- Projected mass derived from the PDI measurements at $10 \mathrm{~mm}$ downstream of the injector is $30-40 \mu \mathrm{m} / \mathrm{mm}^{2}$ compared with $15-20 \mu \mathrm{m} / \mathrm{mm}^{2}$ for USAXS. Given that the PDI requires accurate droplet counting rates, sample volume determination and accurate drop sizing along with data rate weighting of the individual measurements along the path, the agreement is considered reasonable.

- Integrated mass values are consistent between injectors at $690 \mathrm{kPa}$ condition and increase from the x-ray measured plane to the PDI measured axial distance, indicating a decrease in spray velocity.

- The measurement of the path integrated drop size (in terms of the D32) indicate good agreement at $10 \mathrm{~mm}$ downstream. The integrated values from both PDI and USAXS is 100-125 $\mu \mathrm{m}$. The exception is for the 345 $\mathrm{kPa}$ case for SN005. Again, this may be due in part to the dynamic nature of the SN005 spray at $345 \mathrm{kPa}$. 
- Complete profiles of $S_{p}$ at $\mathrm{Z}=10 \mathrm{~mm}$ allowed for a more thorough comparison to two derivations of path specific surface area from PDI measurements. All three profiles reflect similar asymmetries and absolute magnitudes, specifically at the centerline, compare well between USAXS $S_{p}$ and that generated from PDI through the surface mean diameter $\left(D_{20}\right)$.

\section{Acknowledgements}

Research presented in this paper was performed at the 7-BM, and 9-ID beamlines at the Advanced Photon Source at Argonne National Laboratory. Use of the APS is supported by the U.S. Department of Energy (DOE) under Contract No. DEAC0206CH11357. The Argonne fuel spray research is sponsored by the DOE Vehicle Technologies Program under the direction of Gurpreet Singh and Michael Weismiller. The support of Advanced Atomization Technologies (Ethan Hanson) is appreciated relative to the provision of the atomizers.

\section{References}

[1] Arthur H Lefebvre and Vincent G McDonell. Atomization and sprays. CRC press, 2017.

[2] A. Kastengren, J. Ilavsky, Juan Pablo Viera, Raul Payri, D.J. Duke, A. Swantek, F. Zak Tilocco, N. Sovis, and C.F. Powell. International Journal of Multiphase Flow, 92:131 - 139, 2017.

[3] K. E. Matusik, B. A. Sforzo, H. J. Seong, D. J. Duke, A. L. Kastengren, J. llavsky, and C. F. Powell. Atomization and Sprays, 2019. Under Review.

[4] Lee G Dodge. Applied Optics, 26(7):1328-1341, 1987.

[5] Vincent G McDonell and Scott Samuelsen. Sensitivity assessment of a phase-doppler interferometer to usercontrolled settings. Liquid Particle Size Measurement Techniques: 2nd Volume. ASTM International, 1990.

[6] VG McDonnell, GS Samuelsen, MR Wang, CH Hong, and WH Lai. Journal of Propulsion and Power, 10(3):402409, 1994.

[7] Michele Bardi, Raul Payri, Louis Marie C. Malbec, Gilles Bruneaux, Lyle M. Pickett, Julien Manin, Tim Bazyn, and Caroline L. Genzale. Atomization and Sprays, 22(10):807-842, 2012.

[8] Scott B Leask, Alice K Li, Vincent G McDonell, and Scott Samuelsen. ASME 2018 International Mechanical Engineering Congress and Exposition, pp. V08AT10A024-V08AT10A024. American Society of Mechanical Engineers, 2018.

[9] Brandon Sforzo, Scott Leask, Alice Li, Aniket Tekawade, Christopher Powell, Vincent McDonell, and Alan Kastengren. AIAA Scitech 2020 Forum, 2020.

[10] Gabriel L. Jacobsohn, Brandon Sforzo, Alan Kastengren, Aniket Tekawade, Christopher F. Powell, Scott B. Leask, Alice K. Li, and Vincent G. McDonell. AIAA Scitech 2021 Forum, 2021.

[11] Alan Kastengren and Christopher F. Powell. Experiments in Fluids, 55(3):1686, Feb 2014.

[12] Alan L. Kastengren, F. Zak Tilocco, Daniel Duke, Christopher F. Powell, Xusheng Zhang, and Seoksu Moon. Atomization and Sprays, 24(3):251-272, 2014.

[13] Jan Ilavsky, Pete R. Jemian, Andrew J. Allen, Fan Zhang, Lyle E. Levine, and Gabrielle G. Long. Journal of Applied Crystallography, 42(3):469-479, Jun 2009.

[14] Jens Als-Nielsen and Des McMorrow. Elements of modern X-ray physics. John Wiley \& Sons, 2011.

[15] CF Powell, D Duke, AL Kastengren, and J llavsky. 25th ILASS-Americas conference, Pittsburgh, PA, 2013. 